\title{
Integración de servicios de referencia digital con la gestión de una biblioteca: experiencias basadas en Koha
}

\author{
Por Nuria Lloret-Romero, Alicia Sellés-Carot, Moisés Mañas-Carbonell y Fernando Canet-Centellas
}

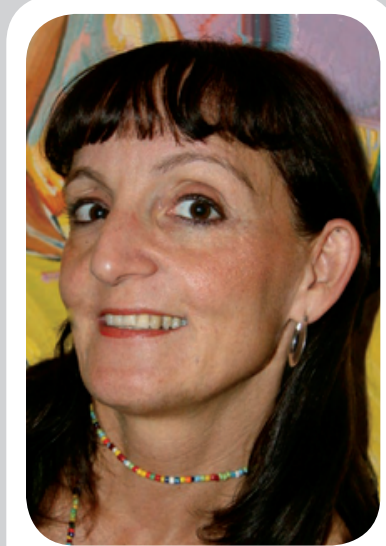

Nuria Lloret-Romero es doctora por la Universidad Politécnica de Valencia (UPV), master en desarrollo de sistemas de información online, soportes magnéticos cd-rom (Masdoc) de la UB, y master en dirección financiera de empresas (Fund. Estudios Bursátiles de Valencia). Profesora titular y directora del Dpto. de Comunicación Audiovisual, Documentación e Historia del Arte (Dcadha) de la UPV. Subdirectora del Inst. de Investigación Univ. IDF y directora del grupo de investigación Calsi. Evaluadora de la UE (programa IST). Socia fundadora de MASmedios, vicepres. de la Fed. Valenciana de empresas de servicios avanzados y asesora del Comité de tecnologías de la información de la Cámara de Comercio de Valencia.

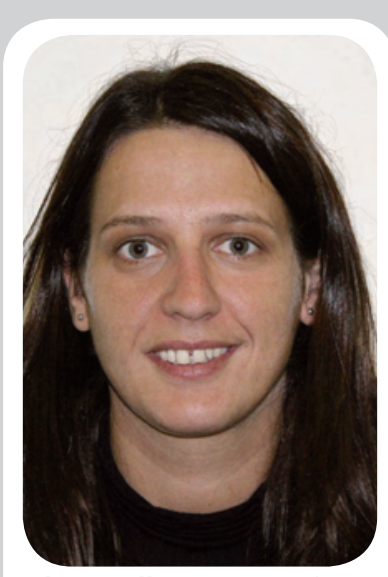

Alicia Sellés-Carot es licenciada en documentación por la Universidad Politécnica de Valencia (UPV) y master en contenidos y aspectos legales en la sociedad de la información. Trabaja en MASmedios desde 2002 y es responsable del área de Servicios Documentales. Ha participado en proyectos relacionados con bibliotecas digitales, digitalización, personalización de interfaces de opac, integración de sistemas de gestión de bibliotecas, gestión documental, repositorios OAI $y$ outsourcing de servicios documentales. Actualmente trabaja en proyectos de $I+D$ relacionados con sistemas open source para bibliotecas (Koha) y la integración de otras aplicaciones open source con sistemas comerciales.

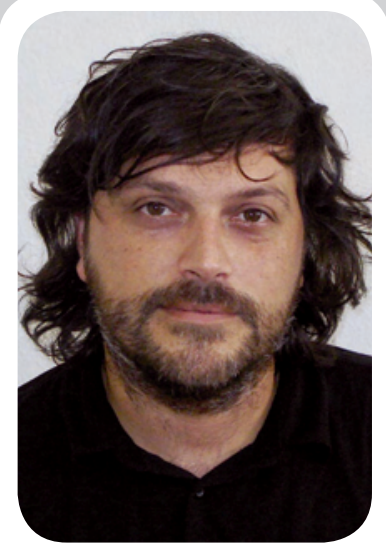

Moisés Mañas-Carbonell es doctor en artes visuales e intermedia y licenciado en bellas artes por la UPV. Especialista universitario en programación de intranets e internet. Es profesor del departamento de escultura (área audiovisuales) de la UPV. Comparte su labor de docente con la dirección creativa, multimedia e I+D en MASmedios desde 1999. Miembro desde 2000 del grupo de investigación I+D+I "Laboratorio de Luz", del Comité técnico del programa del congreso internacional ACE (Internacional conference on advances in computer entertainment technology ACMSIGCHI) entre 2005 y 2008, y del comité artístico del DIMEATS, 2006 (Digital Interactive Media Entertainment \& Arts). http://www.moisesmanas.com

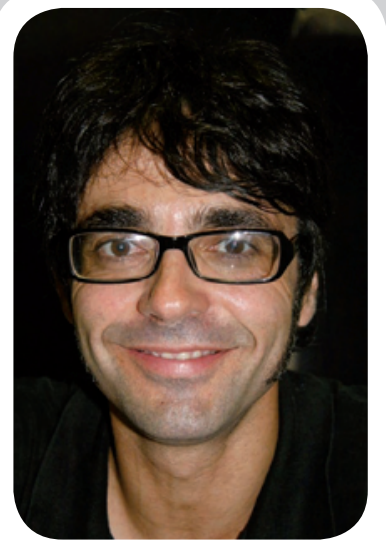

Fernando Canet-Centellas es doctor en comunicación audiovisual y postgrado en herramientas de autor para títulos multimedia por la Universidad Politécnica de Valencia (UPV). Profesor titular de comunicación audiovisual en la UPV, ha participado en numerosos proyectos de investigación. Su principal línea de investigación versa sobre nuevas formas narrativas en el audiovisual contemporáneo en entornos digitales. Es realizador de relatos audiovisuales y ha sido jurado en diferentes festivales de cine. Dirige un programa de televisión en Gandia Televisión y en la televisión de la universidad. Ha sido subdirector docente de su departamento y director del Área de Actividades Culturales de la UPV.

Resumen: El entorno digital y las tecnologías de la información y comunicación abren nuevas vías para la prestación de servicios bibliotecarios, entre ellos el servicio de referencia, cuya misión principal es la resolución de las dudas o necesidades de información de los usuarios. La puesta en marcha del servicio de referencia en este nuevo medio obliga al rediseño de políticas, procedimientos y recursos destinados a ello y por otro lado, requiere el análisis de los servicios y funciones de las aplicaciones informáticas de las que se dispone. Los sistemas integrados de gestión de bibliotecas deben dar respuesta a las necesidades de puesta en marcha de este tipo de servicios. Se presentan los resultados obtenidos en un análisis realizado sobre varios ejemplos de experiencias de nuevos servicios de referencia integrados en un sistema de gestión de bibliotecas (Koha) que no sólo facilitan la prestación de los mismos, sino que también amplían las posibilidades de explotación de los datos para su mejora y evolución.

Palabras clave: Servicios de referencia, Software open source, Sistemas de gestión de bibliotecas, 2.0, Koha.

Title: Integrating digital reference services into library management: experiences based on Koha

Abstract: The digital environment and information and communication technologies open up new ways of providing library services, including the reference service, the principal aim of which is to address users' queries and information needs. Putting the reference service in place in this new environment requires redesigning the policies, procedures and resources applied to it and therefore an analysis of the services and functions of the computing tools available must be undertaken. Integrated library management systems which involve multiple functionalities for delivering services, or which expand the capacity for their delivery, must also respond to the requirements for putting this type of service in place. This article sets out the results obtained in a study carried out on several examples of integrating new reference services into a 
library management system (Koha), which not only enables these services to be delivered but also expands the options for using the data for their improvement and development.

Keywords: Reference services, Open source software, Library management systems, 2.0, Koha.

Lloret-Romero, Nuria; Sellés-Carot, Alicia; Mañas-Carbonell, Moisés; Canet-Centellas, Fernando. "Integración de servicios de referencia digital con la gestión de una biblioteca: experiencias basadas en Koha". El profesional de la información, 2009, noviembre-diciembre, v. 18, n. 6, pp. 654-659.

DOI: 10.3145/epi.2009.nov.09

\section{Introducción}

\section{LA PUESTA EN MARCHA} de servicios de referencia digital requiere la reconsideración de las políticas tradicionales y la adaptación al entorno digital de los servicios existentes, con las implicaciones de uso, gestión y nuevas prestaciones para servicios en la Red.

Este nuevo medio abre un gran abanico de posibilidades para un servicio cuya finalidad principal es la respuesta a las necesidades de información de los usuarios. En las bibliotecas tradicionales el servicio de referencia no es simplemente informativo. También es formativo y de orientación puesto que debe fomentar la capacidad de informarse de los usuarios.

\section{"Un SIGB debe dar respuesta a la gestión del conocimiento corporativo así como a la gestión de la colección"}

Cuando se trata de implementar un sistema integrado de gestión de bibliotecas (SIGB), y además poner en marcha o mejorar otros servicios de información, como el de referencia digital, la flexibilidad del software open source Koha facilita el trabajo. Por ello, el propósito de nuestro análisis no se ha centrado en la variedad de iniciativas existentes para servicios de referencia online, sino que hemos tratado de identificar los puntos clave del entorno digital, los fines del servicio y las posibilidades de ampliación e integración desde aplicaciones globales open source y en particular con Koha.

\section{Servicio de referencia digital}

Tiene los mismos fines y requerimientos que el tradicional, es decir, responder a las necesidades puntuales de información de los usuarios, las cuales pueden ser prácticas (de la propia biblioteca, de la comunidad, del uso de recursos, etc.) o bibliográficas, pero también de orientación y recomendación, de actualidad, etc.

La planificación del servicio tradicional debe tener en cuenta tanto la información como los usuarios y los recursos o fuentes de información disponibles. En el entorno digital habrá además que aprovechar y explotar las ventajas que el nuevo medio presenta, no sólo en cuanto a barreras espacio temporales y formas de comunicación sino también en cuanto a la retroalimentación, la interactividad y la colaboración.

En la bibliografía especializada encontramos descripciones de multitud de iniciativas de servicios de referencia digitales basados en nuevas vías y tecnologías de comunicación entre usuarios y bibliotecarios, así como diferentes clasificaciones en función de la forma de comunicación empleada. Seoane y Barrero (2008) distinguen entre servicios de referencia por voz y por escrito. También Merlo-Vega (2005), que describe diferentes proyectos a ni- vel internacional, menciona como una de las principales propiedades los sistemas telemáticos empleados para que los usuarios realicen sus consultas: chat, formulario (interfaz para envío de mensajes) u otras formas de comunicación.

La gran mayoría se plantean únicamente como servicios informativos mediante nuevas vías de comunicación entre el bibliotecario y el usuario. Pero tanto el servicio de referencia digital como el tradicional deben ir más allá y ser también formativos y orientativos. Es decir, los sistemas digitales deberían planificarse además para asesorar y anticiparse a las necesidades de los usuarios.

\section{Web 2.0 y servicios de referencia}

Los puntos clave en la relación entre profesional y usuario en un entorno web 2.0 no son únicamente la ampliación de las formas de comunicación o la desaparición de las barreras físicas y temporales. Hay que tener en cuenta también la ampliación de los tipos de usuarios y de sus necesidades informativas así como sus cambios de actitud (más colaborativa y social), el uso que realizan de los recursos, cada vez con mayores requerimientos de personalización, de intercambio, de acceso inmediato, etc.

Una de las piezas clave en el sistema tradicional es el bibliotecario referencista, un intermediario entre la información y el usuario. En el entorno 2.0, esta relación ya no es cara a cara, sino desde el sitio web de la biblioteca, por lo que las exi- 


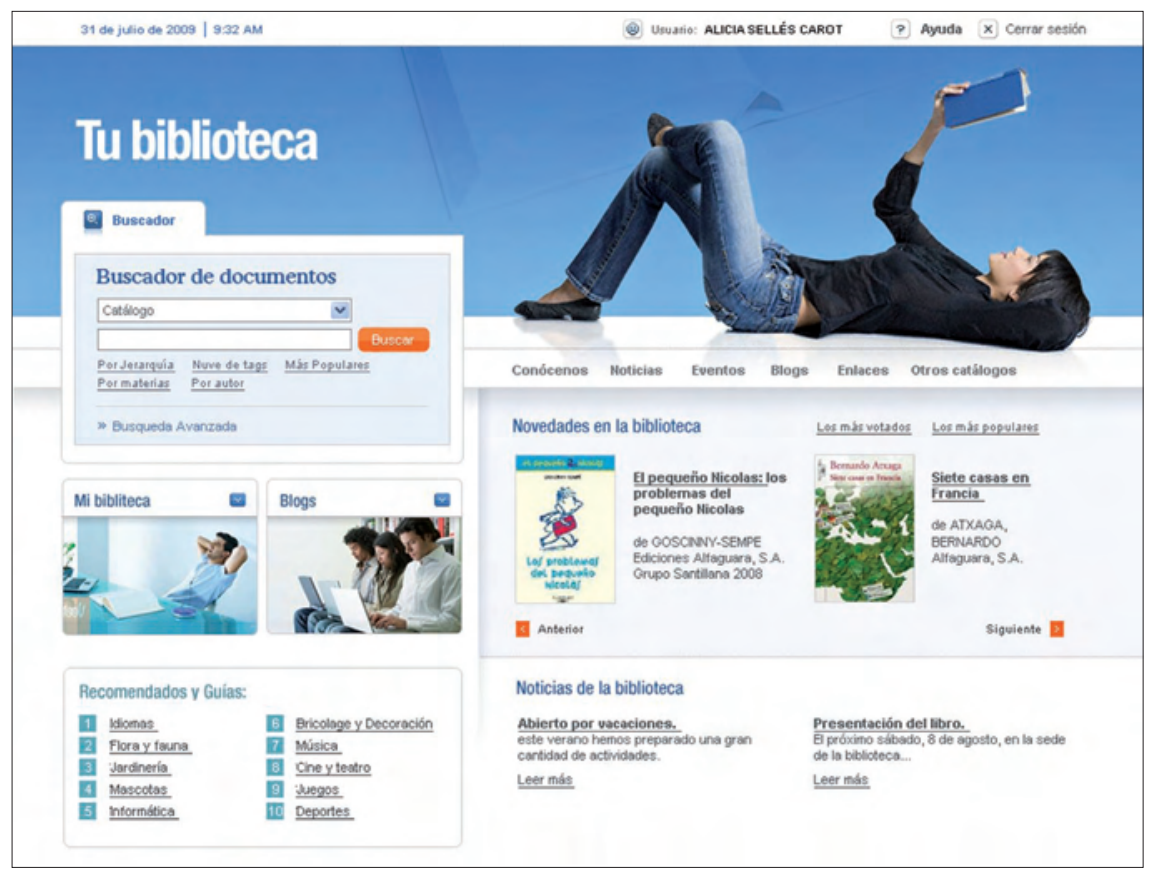

Figura 1. Propuesta de home personalizada en Koha biblioteca es la sede de sus servicios online, donde amplía su labor informativa y de servicio. Por tanto, el sitio web y los servicios que ofrece deben incluirse e integrarse en los procesos técnicos bibliotecarios.

Saorín-Pérez y González-Lorca (2003) identifican el opac como una "primera generación de sistemas de información públicos de la biblioteca, cuya función se amplía y revisa en los portales bibliotecarios, los cuales constituyen la segunda generación".

El opac se ha integrado en los sistemas de gestión de bibliotecas como un módulo junto a los de trabajo técnico, como adquisiciones, catalogación, circulación, etc. Alguno de ellos ha sido remodelado para ofrecer y gestionar servicios digitales, como bibliotecas digitales, pasarelas a servicios comerciales y proveedores de contenidos digitales. Pero también es cierto que la rápida evolución de la tecnología y su implantación en otros medios ha sobrepasado las posibilidades de los SIGB, y en la gran mayoría de las webs de bibliotecas con cierta entidad encontramos integración de varias funciones y aplicaciones, aunque no podemos hablar de siste- mas integrados.

La integración es fundamental en la automatización de bibliotecas, pero ésta debe ir más allá en el contexto digital de manera que se facilite la prestación de servicios, sin que constituya una duplicidad de datos, esfuerzos y recursos.

Esta necesidad de integración pasa por lo que se denomina SIGB extendido (Saorín-Pérez; González-Lorca, 2003), es decir, un sistema integrado de gestión de bibliotecas que ofrezca soluciones a los entornos orgánicos y funcionales del centro, es decir a su estructura organizativa, a sus actividades, y a sus procesos sobre información estructurada y no estructurada. Por tanto, un SIGB debe dar respuesta a la gestión del conocimiento corporativo, a la gestión de la colección, al diseño de servicios y productos y a la gestión de sitios web de servicio o diseño de espacios de atención al usuario.

\section{"Koha ha mejorado sus opacs orientándolos a la colaboración y la personalización, e incluyendo funciones de SIGB extendido"}

\section{Software open source Koha, funciones para un servicio de referencia digital 2.0}

El servicio de referencia digital basado en la web 2.0 debe ser un sistema diseñado para facilitar el acceso y el uso de la información, formado por las personas que atienden el servicio, los usuarios, la información y la tecnología que permite ofrecerlo en red.

Muchos servicios de referencia digital, independientemente de vicio físico, pero también un luga y servicio en la Red. La web de la 
la política institucional y de la implicación para adaptarse al nuevo entorno, se ofrecen sólo de manera parcial o limitada debido al uso de aplicaciones o productos cuya integración resulta complicada o requiere gran esfuerzo.

Koha es un sistema integrado de gestión de bibliotecas open source (código abierto) que funciona con una arquitectura cliente servidor, $\mathrm{y}$ que proporciona flexibilidad $\mathrm{y}$ oportunidades para implementaciones personalizadas. Hace posible la realización de todos los procesos bibliotecarios y su funcionamiento se gestiona vía web con dos tipos de interfaces totalmente independientes: el opac (portal de acceso público) y la interfaz de trabajo técnico bibliotecario (intranet o administración).

La base de muchos de los servicios o actividades que se plantean desde el servicio de referencia es el propio catálogo de la biblioteca. Por ello, Koha ha mejorado las funciones de sus opacs orientándolos a la colaboración y la personalización, e incluyendo algunas de SIGB extendido.

En la versión estable 3.0 de Koha ya encontramos algunas funciones relacionadas con los servicios de referencia, entre ellas:

- RSS: puede utilizarse para la difusión selectiva de información. Koha hace posible la sindicación de las consultas y la recepción de alertas cuando se agrega al catálogo un nuevo registro o ítem que se ajusta a los términos de una búsqueda.

- Listas (públicas y privadas): Koha permite trabajar con listas públicas (de acceso y visibilidad para todos los usuarios) y privadas (de uso particular para quien las genera). De este modo los usuarios pueden participar y realizar listas recomendadas para uso de otros. Esta función se encuentra tanto en la parte pública (opac/portal) como en la interna, para los bibliotecarios. Las listas de libros son interesantes para que tanto usuarios como personal de referencia puedan generar guías de lectura o recomendaciones.

- Registros bibliográficos enriquecidos: el asesoramiento al usuario es otro servicio de referencia que puede ser prestado de forma automática utilizando recursos externos y relacionándolos con los registros que figuran en el catálogo. En este asesoramiento participan el personal de referencia y los usuarios, por lo que la información añadida al registro puede ser de gran utilidad. Koha no sólo hace posible que los usuarios realicen comentarios sobre los registros, sino que además utiliza varias apis (Amazon, Google bo$o k s$, etc.) que son muy sencillas de configurar desde la administración del sistema, para incorporar y relacionar el registro bibliográfico con datos del mismo en Amazon o Google books. Lo más destacado desde el punto de vista del servicio de referencia es la capacidad de importar los datos de registros de Amazon y visualizarlos en la propia ficha del documento, sin enlaces ni ventanas externas, incluyendo las reseñas de los usuarios, los documentos similares y el enlace a Search inside the book para que quien lo desee pueda revisar parte de su contenido.

- Mensajería (noticias, eventos): desde Koha se pueden crear noticias o eventos, que se publican en el opac, pero además el usuario puede configurar la recepción de notificaciones (vía mail o sms) para estos eventos. De este modo el usuario, que sólo ha sido dado de alta una vez en el SIGB, puede obtener información y contenidos de la biblioteca.

Como comentábamos anteriormente, Koha permite la implementación de nuevas funciones atendiendo a las peculiaridades de cada proyecto sin demasiada complejidad. En nuestro análisis sobre Koha hemos incluido en la aplicación nuevos servicios y funciones con el fin de proporcionar información, asesorar y orientar al usuario desde una única plataforma para el personal de la biblioteca. Se trata no sólo de nuevos servicios para el usuario, sino también de facilitar al profesional la prestación de los mismos, pues se integran las funciones básicas de un sistema de gestión de bibliotecas con otras tradicionales para adecuar y ampliar los servicios en el entorno digital.

Entre todas ellas, podemos destacar:

- Valoraciones: a la función de añadir comentarios que incluye la aplicación, se ha incorporado la posibilidad de valorar un registro de manera que de un modo sencillo, sin tener que redactar ningún comentario, se pueda participar y que esta participación anónima sirva de orientación al resto.

\section{"Koha utiliza varias apis (Amazon, Google books, etc.) que integra a la información del registro"}

- Recomendaciones: un usuario del opac puede enviar un registro a terceros. Simplemente se ha habilitado un formulario que envía los datos del registro por email a las direcciones de correo que se indiquen junto con el comentario que se quiera añadir.

- Alertas para autoridades: con la misma filosofía del RSS y de la difusión selectiva de la información, cuando el usuario realiza una consulta desde la búsqueda por autoridades o desde las autoridades de cualquiera de los registros, en la página de resultados puede suscribirse a la autoridad correspondiente. Recibirá un mensaje de alerta cada vez que se incorpore un nuevo registro relacionado con ella. Las 


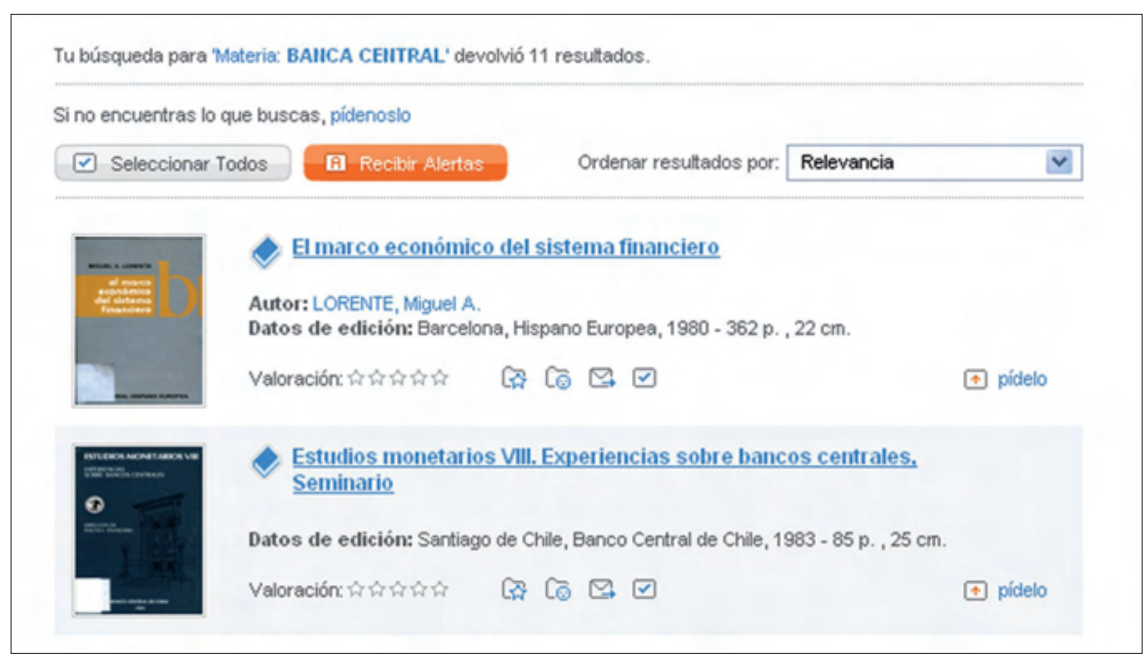

Figura 2. Propuesta de interfaz personalizada para servicio de alertas en Koha

alertas pueden modificarse o darse de baja en cualquier momento.

- Novedades, populares, valorados: la participación del usuario y el uso de sus datos de gestión de los registros, permiten mostrar en el opac más información y de manera más atractiva en las tareas de orientación y referencia. A modo de tablón de anuncios, pueden mostrarse las novedades en el catálogo, automáticamente, sólo con catalogar un nuevo documento sin necesidad de utilizar un gestor de contenidos aparte de la aplicación. Con este sistema podemos difundir de forma inmediata los libros más populares o los mejor valorados. Mención especial merecen los documentos más populares, puesto que en la mayoría de los sistemas de gestión de bibliotecas, éstos son equiparados a los documentos más prestados. En el entorno digital podemos y debemos ampliar esta comparación, y el cálculo que ofrece los registros más populares debe tener en cuenta otras acciones propias del entorno. Es decir, hay documentos digitales que se escapan del módulo de circulación del sistema y además del préstamo existen acciones, en Koha especialmente, que indican que un registro ha sido usado, como las recomendaciones, los comentarios, las valoraciones, la inclusión en las listas, la visualización del registro, etc.
- Redes sociales: esta función facilita la orientación al usuario en cuanto a los documentos que le pueden interesar según sus preferencias. Explotar los datos de los usuarios para relacionarlos entre ellos resulta interesante sobre todo si la información que se muestra es del uso que hacen de la colección. En este sentido, y siempre con su consentimiento, la aplicación puede mostrar usuarios similares y sus datos de interactuación con el sistema, es decir, qué libros saca en préstamo, qué listas tiene, cuáles son sus favoritos, a qué alertas está suscrito...

- Gestión de contenidos: no suele estar integrada en el módulo del opac. En Koha, salvo los eventos y noticias, tampoco está integrada. Aumentar e integrar las funciones en la gestión de contenidos en el módulo opac y en la parte de administración del sistema permite mejorar la información y la forma de mostrarla, y agilizar la prestación de servicios, entre ellos el de referencia. En Koha hemos creado un gestor de contenidos básico que explota los datos del sistema de una forma global y genera contenidos para el portal/opac. Algunos de ellos están relacionados con el servicio de referencia digital y nos gustaría destacarlos. Por ejemplo, la generación de boletines (genéricos o temáticos) con las noticias, los eventos, las novedades de la colección (últimos documentos catalogados) y la posibilidad de incluir en ellos reseñas y recomendaciones de registros existentes en el catálogo (remitiendo allí al usuario para que pueda reservarlo, añadirlo a sus listas, etc.) simplemente realizando una búsqueda en el catálogo y seleccionándolos para su inclusión en el boletín.

Otra cuestión interesante es la gestión dinámica de los contenidos de la ayuda, que puede incluir información del uso del opac, información genérica del uso de la colección, de un servicio o de los recursos disponibles en línea, y las preguntas o dificultades planteadas en los formularios de ayuda y contacto de la web, etc. Por último, una función más avanzada y que exige además bastante implicación en la generación de contenidos, es la creación de glosarios que, siguiendo la filosofía de la wiki y de obras de referencia digitales, relaciona definiciones de términos con registros en el catálogo.

\section{"Koha tiene en cuenta las peculiaridades de cada proyecto sin demasiada complejidad, dando una personalización completa al sitio web"}

\section{Conclusiones}

Los servicios de información y referencia digital no deben plantearse únicamente como la ampliación de las vías de comunicación con el usuario en el entorno digital. La evolución de la tecnología, de las necesidades de los usuarios y de la propia concepción del servicio en este medio exige la integración real de las aplicaciones y recursos disponibles en las bibliotecas. 
La web/opac es la sede digital de los servicios y recursos de la biblioteca, en la que los usuarios interactúan y se comunican $y$, por otro lado, el SIGB es la herramienta principal de realización y automatización de los procesos técnicos profesionales. Los SIGB tradicionales ya presentan múltiples funciones para la prestación de algunos de los servicios bibliotecarios o amplían la capacidad de prestación de los mismos, pero deben seguir evolucionando y conseguir una integración total, para dar respuesta a las necesidades de los usuarios y para rentabilizar esfuerzos y recursos.

Para la puesta en marcha de los servicios de referencia digital, los sistemas integrados contienen y gestionan datos (de usuarios, de la colección, etc.) que deberían explotarse y rentabilizarse para mejorar la información directa que la biblioteca puede ofrecer y el usuario puede pedir, y también para tareas de orientación y recomendación, personalización y respuesta a necesidades concretas, la difusión selectiva de la información, etc.
La utilización de software libre, en este caso Koha, facilita la integración real de aplicaciones, tecnologías y recursos internos y externos, y además aumenta la capacidad de desarrollo para la prestación de servicios e incorporación de nuevas funciones (orientadas al usuario y al personal bibliotecario).

\section{Bibliografía}

García-Delgado, Purificación. "El servicio de referencia digital". El profesional de la información, 2003, v. 12, n. 4, pp. 320-330, ISSN 1386-6710. http://www.elprofesionaldelainformacion.com/ contenidos/2003/julio/11.pdf

International Federation of Library Associations and Institutions (IFLA). Recomendaciones para el servicio de referencia digital.

http://www.ifla.org/VII/s36/pubs/drgO3-s.htm

Manso-Rodríguez, Ramón-Alberto. "Servicio de referencia virtual: teoría y práctica en torno a las políticas para su gestión y desarrollo". Revista española de documentación científica, 2008, v. 31, n. 1, enero-marzo, pp. 39-51, ISSN 0210-0614.

http://redc.revistas.csic.es/index.php/redc/article/view/411/423

Merlo-Vega, José-Antonio. "Servicios públicos de referencia en línea". BiD: textos universitaris de biblioteconomia i documentació, 2005, n. 14, jun.

http://www.ub.es/bid/14merlo2.htm

Merlo-Vega, José-Antonio. La calidad de la información telemática: evaluación de servicios públicos de referencia digital. Tesis doctoral dir- igida por José-Antonio Frías-Montoya. Universidad de Salamanca, 2008.

http://gredos.usal.es:800/iii_/handle?recid= d10444762

Merlo-Vega, José-Antonio. "Referencia digital y servicios electrónicos de información". Аnuario ThinkEPI 2009, pp. 137-140, ISSN 18866344.

O'Reilly, Tim. What is Web 2.0. Design patterns and business models for the next generation of software. 2005.

http://www.oreillynet.com/lpt/a/6228

Saorín-Pérez, Tomás; González-Lorca, Jesús. "Dentro de los portales bibliotecarios: flujo de trabajo en la automatización de bibliotecas". Scire: Representación y organización del conocimiento, 2003, v. 9, n. 2, pp. 37-59, ISSN 1135-3716.

http://ibersid.eu/ojs/index.php/scire/article/ view/1466/1444

Seoane-García, Catuxa; Barrero-Robledo, Vanesa. "La evolución de los servicios de referencia digitales en la Web 2.0". En: VIII Congreso de Anabad, Madrid, 13-15 febrero 2008. http://eprints.rclis.org/15085/1/servicios-de-referencia-en-la-web-20.pdf

Nuria Lloret-Romero, Moisés Mañas-Carbonell y Fernando CanetCentellas. Universidad Politécnica de Valencia nlloret@upvnet.upv.es moimacar@upvnet.upv.es fercacen@upvnet.upv.es

\section{Alicia Sellés-Carot. MASmedios alicia@masmedios.com}

\section{(ii) soft Software Vigilancia Tecnológica}

\section{Recuperación y gestión} de la información 